DOI: https://doi.org/10.14311/TPFM.2022.009

\title{
THE HYDROSTATIC APPROXIMATION OF COMPRESSIBLE ANISOTROPIC NAVIER-STOKES EQUATIONS
}

\author{
H. Gao ${ }^{1}$, Š. Nečasová ${ }^{2}$, T. Tang ${ }^{3}$
}

${ }^{1}$ School of Mathematical Sciences, Southeast University, Nanjing 211189, P.R. China

${ }^{2}$ Institute of Mathematics of the Academy of Sciences of the Czech Republic, Žitná 25, 11567, Praha 1, Czech Republic

${ }^{3}$ School of Mathematical Science, Yangzhou University, Yangzhou 225002, P. R. China

\begin{abstract}
The aim of the paper is to give a rigorous derivation of the hydrostatic approximation by taking the small aspect ratio limit to the Navier-Stokes equations. The aspect ratio (the ratio of the depth to horizontal width) is a geometrical constraint in the general large scale geophysical motions meaning that the vertical scale is significantly smaller than horizontal.
\end{abstract}

Keywords: anisotropic Naiver-Stokes equations, aspect ratio limit, hydrostatic approximation, compressible Primitive Equations.

\section{Introduction}

The study of behavior of the atmosphere and ocean have attracted attention in the scientific research community. To model the motion and state of the atmosphere the equations of compressible motion is used. In such model the vertical scale of the atmosphere is much more smaller than the planetary horizontal scale. Therefore, many scientists have suggested the viscosity coefficients must be anisotropic, such as $[4,19,21]$. The anisotropic Navier-Stokes equations are widely used in geophysical fluid dynamics. In this paper, we consider the following compressible anisotropic Navier-Stokes equations

$$
\left\{\begin{array}{l}
\partial_{t} \rho+\operatorname{div}(\rho \mathbf{u})=0 \\
(\rho \mathbf{u})_{t}+\operatorname{div}(\rho \mathbf{u} \otimes \mathbf{u})+\nabla p(\rho)=\mu_{x} \Delta_{x} \mathbf{u}+\mu_{z} \partial_{z z} \mathbf{u}
\end{array}\right.
$$

in the thin domain $(0, T) \times \Omega_{\epsilon}$. Here $\Omega_{\epsilon}=\left\{(x, z) \mid x \in \mathbb{T}^{2},-\epsilon<z<\epsilon\right\}, x$ denotes the horizontal direction and $z$ denotes the vertical direction, while, $\mu_{x}$ and $\mu_{z}$ are given constant horizontal viscous coefficient and vertical viscous coefficient. The velocity $\mathbf{u}=(\mathbf{v}, w)$, where $\mathbf{v}(t, x, z) \in \mathbb{R}^{2}$ and $w(t, x, z) \in \mathbb{R}$ represent the horizonal velocity and vertical velocity, respectively. Through out this paper, we use $\operatorname{div} \mathbf{u}=\operatorname{div}_{\mathrm{x}} \mathbf{v}+\partial_{\mathrm{z}} \mathrm{w}$ and $\nabla=\left(\nabla_{x}, \partial_{z}\right)$ to denote the three-dimensional spatial divergence and gradient respectively, and $\Delta_{x}$ stands for horizontal Laplacian. As atmosphere and ocean are the thin layers, where the fluid layer depth is small compared to radius of sphere, Pedlosky [19] pointed out that "the pressure difference between any two points on the same vertical line depends only on the weight of the fluid between these points...". Here we neglect the gravity and suppose the pressure $p(\rho)$ satisfies the barotropic pressure law where the pressure and the density are related by the formula: $p(\rho)=\rho^{\gamma}(\gamma>1)$. Therefore we assume the density $\rho$ is independent of $z$ that is $\rho=\rho(t, x)$. This plausible assumption agrees well with experiment and is frequently taken as a hypothesis in geophysical fluid dynamics.

Similar to the assumptions by $[1,14]$, we suppose $\mu_{x}=1$ and $\mu_{z}=\epsilon^{2}$. As stressed by Azérad and Guillén [1], it is necessary to consider the above anisotropic viscosities scaling, which is fundamental for the derivation of Primitive Equations (PE). ${ }^{1}$

${ }^{1}$ For completeness we will write the system $(\mathrm{PE})$ :

$$
\left\{\begin{array}{l}
\partial_{t} \mathbf{v}+\operatorname{div}_{x}(\mathbf{v} \otimes \mathbf{v})+\partial_{z}(\mathbf{v} w)+\nabla_{x} p=\Delta_{x} \mathbf{v}+\partial_{z z} \mathbf{v} \\
\partial_{z} p=0 \\
\operatorname{div}_{x} \mathbf{v}+\partial_{z} w=0
\end{array}\right.
$$


Under this assumption, the system is rewritten as the following

$$
\left\{\begin{array}{l}
\partial_{t} \rho+\operatorname{div}_{x}(\rho \mathbf{v})+\partial_{z}(\rho w)=0 \\
\rho \partial_{t} \mathbf{v}+\rho(\mathbf{u} \cdot \nabla) \mathbf{v}-\Delta_{x} \mathbf{v}-\epsilon^{2} \partial_{z z} \mathbf{v}+\nabla_{x} p(\rho)=0 \\
\rho \partial_{t} w+\rho \mathbf{u} \cdot \nabla w-\Delta_{x} \mathbf{w}-\epsilon^{2} \partial_{z z} w+\partial_{z} p(\rho)=0
\end{array}\right.
$$

Inspired by $[1,14]$, we introduce the following new unknowns,

$$
\mathbf{u}_{\epsilon}=\left(\mathbf{v}_{\epsilon}, w_{\epsilon}\right), \mathbf{v}_{\epsilon}(x, z, t)=\mathbf{v}(x, \epsilon z, t), w_{\epsilon}=\frac{1}{\epsilon} w(x, \epsilon z, t), \rho_{\epsilon}=\rho(x, t),
$$

for any $(x, z) \in \Omega:=\mathbb{T}^{2} \times(-1,1)$. Then the system (1.3) becomes the following compressible scaled Navier-Stokes equations (CNS):

$$
\left\{\begin{array}{l}
\partial_{t} \rho_{\epsilon}+\operatorname{div}_{x}\left(\rho_{\epsilon} \mathbf{v}_{\epsilon}\right)+\partial_{z}\left(\rho_{\epsilon} w_{\epsilon}\right)=0 \\
\rho_{\epsilon} \partial_{t} \mathbf{v}_{\epsilon}+\rho_{\epsilon}\left(\mathbf{u}_{\epsilon} \cdot \nabla\right) \mathbf{v}_{\epsilon}-\Delta_{x} \mathbf{v}_{\epsilon}-\partial_{z z} \mathbf{v}_{\epsilon}+\nabla_{x} p\left(\rho_{\epsilon}\right)=0, \\
\epsilon^{2}\left(\rho_{\epsilon} \partial_{t} w_{\epsilon}+\rho_{\epsilon} \mathbf{u}_{\epsilon} \cdot \nabla w_{\epsilon}-\Delta_{x} \mathbf{w}_{\epsilon}-\partial_{z z} w_{\epsilon}\right)+\partial_{z} p\left(\rho_{\epsilon}\right)=0 .
\end{array}\right.
$$

We supplement the CNS with the following boundary and initial conditions ${ }^{2}$ :

$$
\begin{gathered}
\rho_{\epsilon}, \mathbf{u}_{\epsilon} \text { are periodic in } x, y, z, \\
\left.\left(\rho_{\epsilon}, \mathbf{u}_{\epsilon}\right)\right|_{t=0}=\left(\rho_{0}, \mathbf{u}_{0}\right) .
\end{gathered}
$$

The goal of this work is to investigate the limit process $\epsilon \rightarrow 0$ in the system of (1.4). Precisely, to show that the system of (1.4) converges in a certain sense to the following compressible Primitive Equations(CPE):

$$
\left\{\begin{array}{l}
\partial_{t} \rho+\operatorname{div}_{x}(\rho \mathbf{v})+\partial_{z}(\rho w)=0 \\
\partial_{t}(\rho \mathbf{v})+\operatorname{div}_{x}(\rho \mathbf{v} \otimes \mathbf{v})+\partial_{z}(\rho \mathbf{v} w)+\nabla_{x} p(\rho)=\Delta_{x} \mathbf{v}+\partial_{z z} \mathbf{v} \\
\partial_{z} p(\rho)=0
\end{array}\right.
$$

Our goal is to rigorously show the limit in the framework of weak solutions of CNS. Recently, Bella, Feireisl and Novotný [2], Maltese and Novotný [18] proved the limit passage from 3D compressible Navier-Stokes equations to 1D and 2D compressible Navier-Stokes equations in thin domain. See also result by Ducomet et al. [6]. Heuristically, inspired by their works, we develop and adapt the corresponding idea of relative entropy inequality for compressible Navier-Stokes equations. There are significant differences of the mathematical structure between Navier-Stokes equations and CPE model. Due to the hydrostatic approximation, there is no information for the vertical velocity in the momentum equation of CPE model, and the vertical velocity is determined by the horizontal velocity via the continuity equation, so it is very difficult to analyze the CPE model. Therefore, the classical method used in Navier-Stokes system can not be applied straightforwardly to CPE. Luckily, based on our previous work [11] of weak-strong uniqueness to CPE, we prove the aspect ratio limit of compressible anisotropic Navier-Stokes equations. This is the first work to use the relative entropy inequality for proving the hydrostatic approximation at the compressible case. For the introduction of the versatile relative entropy inequality, see [10]. Last but not least, let us mention that the corner-stone analysis of our results is based on the relative energy inequality which was invented by Dafermos, see [5] and by Germain,[13], who introduced it into compressible Navier-Stokes equations. After that Feireisl and his co-authors $[7,8,9]$ generalized the relative energy inequality and applied such inequality for solving various compressible fluid model problems.

\section{Main result}

Before stating our main result, we give the definition of a weak solution for CNS and a strong solution for CPE. Recently, Bresch and Jabin [3] introduced different compactness method from

\footnotetext{
${ }^{2}$ Let us mention that explanation of the periodicity boundary conditions for compressible PE can be found in paper by Liu and Titi-arxiv:1905.09367, Page 4, line 14 .
} 
Lions or Feireisl which can be applied to anisotropical stress tensor. ${ }^{3}$ Bresch and Jabin has proved the global existence of weak solutions for non-monotone pressure and for anisotropical stress tensor. Therefore, following their theory, we can obtain the existence of weak solution for monotone pressure and anisotropic viscous stress tensor. Let us recall their definitions here.

\subsection{Dissipative weak solutions of CNS}

Definition 2.1 We say that $\left[\rho_{\epsilon}, \mathbf{u}_{\epsilon}\right]$ with $\mathbf{u}_{\epsilon}=\left(\mathbf{v}_{\epsilon}, w_{\epsilon}\right)$ is a finite energy weak solution to the system of (1.4), supplemented with initial data (1.5) if $\rho_{\epsilon}=\rho_{\epsilon}(x, t)$ and

$$
\begin{aligned}
& \mathbf{u}_{\epsilon} \in L^{2}\left(0, T ; H^{1}(\Omega)\right), \rho\left|\mathbf{u}_{\epsilon}\right|^{2} \in L^{\infty}\left(0, T ; L^{1}(\Omega)\right), \\
& \rho_{\epsilon} \in L^{\infty}\left(0, T ; L^{\gamma}(\Omega)\right) \cap C\left([0, T], L^{1}(\Omega)\right), \rho_{\epsilon} \geq 0, \gamma>4,
\end{aligned}
$$

- the continuity equation

$$
\left[\int_{\Omega} \rho_{\epsilon} \psi d x d z\right]_{t=0}^{t=\tau}=\int_{0}^{\tau} \int_{\Omega} \rho_{\epsilon} \partial_{t} \psi+\rho_{\epsilon} \mathbf{v}_{\epsilon} \cdot \nabla_{x} \psi+\rho_{\epsilon} w_{\epsilon} \partial_{z} \psi d x d z d t
$$

holds for all $\psi \in C_{c}^{\infty}([0, T) \times \bar{\Omega})$;

- the momentum equation

$$
\begin{array}{r}
{\left[\int_{\Omega} \rho_{\epsilon} \mathbf{v}_{\epsilon} \varphi_{\mathbf{H}} d x d z\right]_{t=0}^{t=\tau}-\int_{0}^{\tau} \int_{\Omega} \rho_{\epsilon} \mathbf{v}_{\epsilon} \partial_{t} \varphi_{\mathbf{H}} d x d z d t-\int_{0}^{\tau} \int_{\Omega} \rho_{\epsilon} \mathbf{u}_{\epsilon} \mathbf{v}_{\epsilon} \cdot \nabla \varphi_{\mathbf{H}} d x d z d t} \\
+\int_{0}^{\tau} \int_{\Omega} \nabla \mathbf{v}_{\epsilon}: \nabla \varphi_{\mathbf{H}} d x d z d t-\int_{0}^{\tau} \int_{\Omega} p\left(\rho_{\epsilon}\right) d i v_{x} \varphi_{\mathbf{H}} d x d z d t=0
\end{array}
$$

and

$$
\begin{aligned}
\epsilon^{2}\left[\int_{\Omega} \rho_{\epsilon} w_{\epsilon} \varphi_{3} d x d z\right]_{t=0}^{t=\tau} & -\epsilon^{2} \int_{0}^{\tau} \int_{\Omega} \rho_{\epsilon} w_{\epsilon} \partial_{t} \varphi_{3} d x d z d t-\epsilon^{2} \int_{0}^{\tau} \int_{\Omega} \rho_{\epsilon} \mathbf{u}_{\epsilon} w_{\epsilon} \cdot \nabla \varphi_{3} d x d z d t \\
& +\epsilon^{2} \int_{0}^{\tau} \int_{\Omega} \nabla w_{\epsilon} \cdot \nabla \varphi_{3} d x d z d t-\int_{0}^{\tau} \int_{\Omega} p\left(\rho_{\epsilon}\right) \partial_{z} \varphi_{3} d x d z d t=0
\end{aligned}
$$

holds for any spatially periodic function $\varphi_{\mathbf{H}}, \varphi_{3} \in C_{c}^{\infty}([0, T) \times \bar{\Omega})$. Combining $(2.3)-(2.4)$, we obtain

$$
\begin{aligned}
& {\left[\int_{\Omega} \rho_{\epsilon} \mathbf{v}_{\epsilon} \varphi_{\mathbf{H}} d x d z+\epsilon^{2} \int_{\Omega} \rho_{\epsilon} w_{\epsilon} \varphi_{3} d x d z\right]_{t=0}^{t=\tau}} \\
& \quad-\int_{0}^{\tau} \int_{\Omega} \rho_{\epsilon} \mathbf{v}_{\epsilon} \partial_{t} \varphi_{\mathbf{H}} d x d z d t-\epsilon^{2} \int_{0}^{\tau} \int_{\Omega} \rho_{\epsilon} w_{\epsilon} \partial_{t} \varphi_{3} d x d z d t \\
& \quad-\int_{0}^{\tau} \int_{\Omega} \rho_{\epsilon} \mathbf{v}_{\epsilon} \otimes \mathbf{v}_{\epsilon}: \nabla_{x} \varphi_{\mathbf{H}} d x d z d t-\int_{0}^{\tau} \int_{\Omega} \rho_{\epsilon} \mathbf{v}_{\epsilon} w_{\epsilon} \cdot \partial_{z} \varphi_{H} d x d z d t \\
& \quad-\epsilon^{2} \int_{0}^{\tau} \int_{\Omega} \rho_{\epsilon} \mathbf{v}_{\epsilon} w_{\epsilon} \cdot \nabla_{x} \varphi_{3} d x d z d t-\epsilon^{2} \int_{0}^{\tau} \int_{\Omega} \rho_{\epsilon} w_{\epsilon}^{2} \partial_{z} \varphi_{3} d x d z d t \\
& \quad+\int_{0}^{\tau} \int_{\Omega} \nabla \mathbf{v}_{\epsilon}: \nabla \varphi_{\mathbf{H}} d x d z d t+\epsilon^{2} \int_{0}^{\tau} \int_{\Omega} \nabla w_{\epsilon} \cdot \nabla \varphi_{3} d x d z d t-\int_{0}^{\tau} \int_{\Omega} p\left(\rho_{\epsilon}\right) d i v \varphi d x d z d t=0
\end{aligned}
$$

where spatially periodic function $\varphi=\left(\varphi_{\mathbf{H}}, \varphi_{3}\right) \in C_{c}^{\infty}([0, T) \times \bar{\Omega})$ and $\operatorname{div} \varphi=\operatorname{div}_{x} \varphi_{\mathbf{H}}+\partial_{z} \varphi_{3}$,

- the energy inequality

$$
\left.\left[\int_{\Omega} \frac{1}{2} \rho_{\epsilon}\left|\mathbf{v}_{\epsilon}\right|^{2}+\frac{\epsilon^{2}}{2} \rho_{\epsilon}\left|w_{\epsilon}\right|^{2}+P\left(\rho_{\epsilon}\right)\right) d x d z\right]\left.\right|_{t=0} ^{t=\tau}+\int_{0}^{\tau} \int_{\Omega}\left(\left|\nabla \mathbf{v}_{\epsilon}\right|^{2}+\epsilon^{2}\left|\nabla w_{\epsilon}\right|^{2}\right) d x d z d t \leq 0
$$

holds for a. a $\tau \in(0, T)$, where $P(\rho)=\rho \int_{1}^{\rho} \frac{p(z)}{z^{2}} d z$.

\footnotetext{
${ }^{3}$ Let us emphasize the result of Bresch and Jabin is valid only for small coefficients of viscosities
} 


\subsection{Strong solution of CPE}

We say that $(r, \mathbf{U}), \mathbf{U}=(\mathbf{V}, W)$ is a strong solution to the CPE system $(1.6)$ in $(0, T) \times \Omega$, if

$$
\begin{aligned}
& r^{\frac{1}{2}} \in L^{\infty}\left(0, T ; H^{2}(\Omega)\right), \partial_{t} r^{\frac{1}{2}} \in L^{\infty}\left(0, T ; H^{1}(\Omega)\right), r>0 \text { for all }(t, x), \\
& \mathbf{V} \in L^{\infty}\left(0, T ; H^{3}(\Omega)\right) \cap L^{2}\left(0, T ; H^{4}(\Omega)\right), \partial_{t} \mathbf{V} \in L^{2}\left(0, T ; H^{2}(\Omega)\right),
\end{aligned}
$$

with initial data $r_{0}^{\frac{1}{2}} \in H^{2}(\Omega), r_{0}>0$ and $\mathbf{V}_{0} \in H^{3}(\Omega)$. Liu and Titi [15] have proved the local existence of strong solution to CPE system (1.6).

Remark 2.1 As the density is independent of $z$, we can obtain the following information of vertical velocity for the weak solution of CNS :

$$
\rho w(x, z, t)=-\operatorname{div}_{\mathbf{x}}(\rho \widetilde{\mathbf{v}})+\operatorname{zdiv}_{\mathbf{x}}(\rho \overline{\mathbf{v}}) \text {, in the sense of } \mathrm{H}^{-1}(\Omega),
$$

where $^{4}$

$$
\widetilde{\mathbf{v}}(x, z, t)=\int_{0}^{z} v(x, s, t) d s, \bar{v}(x, t)=\int_{0}^{1} v(x, z, t) d z .
$$

Similarly, we can obtain the same equation for the strong solution of CPE in the classical sense. There is no information about $w$, so we need to derive its information. We should emphasize that (2.7) is the key step to obtain the existence of weak solution for CPE in [16, 20], which is inspired by incompressible case.

\subsection{Versatile relative entropy inequality}

Motivated by $[7,8]$, for any finite energy weak solution $(\rho, \mathbf{u})$, where $\mathbf{u}=(\mathbf{v}, w)$, to the CNS system, we introduce the relative energy functional

$$
\begin{aligned}
\mathcal{E}(\rho, \mathbf{u} \mid r, \mathbf{U}) & =\int_{\Omega}\left[\frac{1}{2} \rho|\mathbf{v}-\mathbf{V}|^{2}+\frac{\epsilon^{2}}{2} \rho|w-W|^{2}+P(\rho)-P^{\prime}(r)(\rho-r)-P(r)\right] d x d z \\
& =\int_{\Omega}\left(\frac{1}{2} \rho|\mathbf{v}|^{2}+\frac{\epsilon^{2}}{2} \rho|w|^{2}+P(\rho)\right) d x d z-\int_{\Omega}\left(\rho \mathbf{v} \cdot \mathbf{V}+\epsilon^{2} \rho w W\right) d x d z \\
& +\int_{\Omega}\left[\rho \frac{|\mathbf{V}|^{2}}{2}+\frac{\epsilon^{2}}{2} \rho|W|^{2}-\rho P^{\prime}(r)\right] d x d z+\int_{\Omega} p(r) d x d z \\
& =\sum_{i=1}^{4} I_{i},
\end{aligned}
$$

where $r>0, \mathbf{U}=(\mathbf{V}, W)$ are smooth "test" functions, $\mathbf{V}, W \in C_{c}^{\infty}([0, T) \times \bar{\Omega})$ are spatially periodic functions. Here we have used $r P^{\prime}(r)-P(r)=p(r)$.

Lemma 2.1 Let $(\rho, \mathbf{v}, w)$ be a dissipative weak solution introduced in Definition 2.1. Then $(\rho, \mathbf{v}, w)$ satisfy the versatile relative entropy inequality

$$
\begin{aligned}
\left.\mathcal{E}(\rho, \mathbf{u} \mid r, \mathbf{U})\right|_{t=0} ^{t=\tau}+\int_{0}^{\tau} \int_{\Omega}\left(\nabla \mathbf{v}:(\nabla \mathbf{v}-\nabla \mathbf{V})+\epsilon^{2}|\nabla w|^{2}\right) d x d z d t \\
\leq \int_{0}^{\tau} \int_{\Omega} \rho\left(\partial_{t} \mathbf{V}+\mathbf{v} \cdot \nabla_{x} \mathbf{V}+w \partial_{z} \mathbf{V}\right)(\mathbf{V}-\mathbf{v}) d x d t \\
\quad+\epsilon^{2} \int_{0}^{\tau} \int_{\Omega} \rho\left(\partial_{t} W+\mathbf{v} \cdot \nabla_{x} W+w \partial_{z} W\right)(W-w) d x d z d t+\epsilon^{2} \int_{0}^{\tau} \int_{\Omega} \nabla w \cdot \nabla W d x d z d t \\
\quad-\int_{0}^{\tau} \int_{\Omega} P^{\prime \prime}(r)\left((\rho-r) \partial_{t} r+\rho \mathbf{v} \cdot \nabla_{x} r\right) d x d z d t-\int_{0}^{\tau} \int_{\Omega} p(r) \operatorname{div}_{x} \mathbf{V} d x d z d t
\end{aligned}
$$

\footnotetext{
${ }^{4}$ The calcuation of $\rho w$ can be seen in details see Liu and Titi [17] page 1920 and see [20], page 4 .
} 


\subsection{Main result}

Now, we are ready to state our main result.

Theorem 2.1 Let $\gamma>4, T_{\max }>0$ be the life time of strong solution to CPE system (1.6) corresponding to initial data $\left[r_{0}, \mathbf{V}_{0}\right]$. Let $\left(\rho_{\epsilon}, \mathbf{u}_{\epsilon}\right), \mathbf{u}_{\epsilon}=\left(\mathbf{v}_{\epsilon}, w_{\epsilon}\right)$ be a sequence of dissipative weak solutions to the CNS system (1.4) from the initial data $\left(\rho_{0, \epsilon}, \mathbf{u}_{0, \epsilon}\right)$. Suppose that $\rho_{0, \epsilon}>0, r_{0}>0$ and

$$
\mathcal{E}\left(\rho_{0, \epsilon}, \mathbf{u}_{0, \epsilon} \mid r_{0}, \mathbf{U}_{0}\right) \rightarrow 0
$$

where $\mathbf{U}_{0}=\left(\mathbf{V}_{0}, W_{0}\right)$, then

$$
\text { ess } \sup _{t \in\left(0, T_{\max }\right)} \mathcal{E}\left(\rho_{\epsilon}, \mathbf{u}_{\epsilon} \mid r, \mathbf{U}\right) \rightarrow 0,
$$

where $\mathbf{U}=(\mathbf{V}, W)$ and the couple $(r, \mathbf{U})$ satisfy the CPE system (1.6) on the time interval $\left[0, T_{\max }\right)$.

For more details, see [10, 12].

\section{Acknowledgment}

We would like to thank to Prof. A. Novotný for his remarks and suggestions during his visit in Prague in the spring 2021. The research of H. G is partially supported by the NSFC Grant No. 12171084. The research of Š.N. is supported by the Czech Sciences Foundation (GAČR), GA19-04243S and RVO 67985840. The research of T.T. is supported by the NSFC Grant No. 11801138.

\section{References}

[1] P. Azérad and F. Guillén: Mathematical justification of the hydrostatic approximation in the primitive equations of geophysical fluid dynamics, SIAM J. Math. Anal., 33 (2001), 847-859.

[2] P. Bella, E. Feireisl and A. Novotný: Dimension reduction for compressible viscous fluids, Acta Appl. Math., 134 (2014), 111-121.

[3] D. Bresch and P.E. Jabin: Global existence of weak solutions for compressible Navier-Stokes equations: thermodynamically unstable pressure and anisotropic viscous stress tensor, Ann. of Math., 188 (2018), 577-684.

[4] K. Bryan: A numerical method for the study of the circulation of the world ocean, J. Comp. Phys., 4 (1969), 347-376

[5] C.M. Dafermos: The second law of thermodynamics and stability, Arch. Rational Mech. Anal., 70 (1979) 167-179.

[6] B. Ducomet, Š. Nečasová, M. Pokorný and M.A. Rodríguez-Bellido: Derivation of the NavierStokes-Poisson system with radiation for an accretion disk, J. Math. Fluid Mech., 20 (2018), 697-719.

[7] E. Feireisl, J.B. Jin and A. Novotný: Relative entropies, suitable weak solutions, and weakstrong uniqueness for the compressible Navier-Stokes system, J. Math. Fluid Mech., 14 (2012), 717-730.

[8] E. Feireisl and A. Novotný: Singular limits in thermodynamics of viscous fluids, Advances in Mathematical Fluid Mechanics, Birkhäuser, Basel, 2009.

[9] E. Feireisl, J.B. Jin and A. Novotný: Inviscid incompressible limits of strongly stratified fluids, Asymptot. Anal., 89 (2014), 307-329.

[10] H.J. Gao, Š. Nečasová and T. Tang: On the hydrostatic approximation of compressible anisotropic Navier-Stokes equations, Comptes Rendus Mathématique, 6 (2021), 639-644. 
[11] H.J. Gao, Š. Nečasová and T. Tang: On weak-strong uniqueness and singular limit for the compressible Primitive Equations, Discrete Contin. Dyn. Syst. Ser. A, 40 (2020), 4287-4305.

[12] H.J. Gao, Š. Nečasová and T. Tang: On the hydrostatic approximation of compressible anisotropic Navier-Stokes equations - rigorous justification, arxiv arXiv:2011.04810

[13] P. Germain: Weak-strong uniqueness for the isentropic compressible Navier-Stokes system, $J$. Math. Fluid Mech., 13 (2011), 137-146.

[14] J.K. Li, and E.S. Titi: The primitive equations as the small aspect ratio limit of the NavierStokes equations: rigorous justification of the hydrostatic approximation, J. Math. Pures Appl., 124 (2019), 30-58.

[15] X. Liu and E.S. Titi: Local well-posedness of strong solutions to the three-dimensional compressible Primitive Equations, arxiv1806.09868v1.

[16] X. Liu and E.S. Titi: Global existence of weak solutions to the compressible Primitive Equations of atmosphereic dynamics with degenerate viscositites, SIAM J. Math. Anal., 51 (2019), 1913-1964.

[17] X. Liu and E.S. Titi: Zero Mach number limit of the compressible Primitive Equations Part I: well-prepared initial data, Arch. Ration. Mech. Anal., 238 (2020), 705-747.

[18] D. Maltese and A. Novotný: Compressible Navier-Stokes equations on thin domains, J. Math. Fluid Mech., 16 (2014), 571-594.

[19] W.M. Pedlosky:Geophysical Fluid Dynamics, Springer-Verlag, 1979.

[20] F.C. Wang, C.S. Dou and Q.S. Jiu: Global weak solutions to 3D compressible primitive equations with density-dependent viscosity, J. Math. Phys. 61 (2020), no. 2, 021507, 33 pp.

[21] W.M. Washington and C.L. Parkinson:An Introduction to Three-Dimensional Climate Modelling, Oxford University Press, 1986. 